November 2002

\title{
Comparisons of American, Israeli, Italian and Mexican physicians and nurses on the total and factor scores of the Jefferson scale of attitudes toward physician-nurse collaborative relationships
}

\author{
Mohammadreza Hojat \\ Thomas Jefferson University \\ Joseph S. Gonnella \\ Thomas Jefferson University \\ Thipmas is. Na Nasca aditional works at: https://jdc.jefferson.edu/crmehc \\ Thomas Jefferson University \\ Part of the Psychiatry Commons \\ pylyia kifiedds
}

\section{Americo Cicchetti \\ Recommended Citation}

Hojat, Mohammadreza; Gonnella, Joseph S.; Nasca, Thomas J.; Fields, Sylvia K.; Cicchetti, Americo; Lo Scalzo, Alessandra; Taroni, Francesco; Vincenza Amicosante, Anna Maria; Macinati,

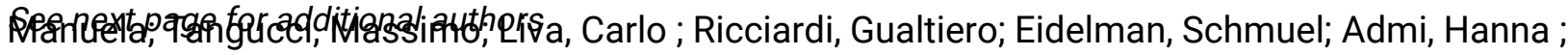
Geva, Hana; Mashiach, Tanya; Alroy, Gideon; Alcorta-Gonzalez, Adelina; Ibarra, David; and TorresRuiz, Antonio, "Comparisons of American, Israeli, Italian and Mexican physicians and nurses on the total and factor scores of the Jefferson scale of attitudes toward physician-nurse collaborative relationships" (2002). CRMEHC Faculty Papers. Paper 3.

https://jdc.jefferson.edu/crmehc/3

This Article is brought to you for free and open access by the Jefferson Digital Commons. The Jefferson Digital Commons is a service of Thomas Jefferson University's Center for Teaching and Learning (CTL). The Commons is a showcase for Jefferson books and journals, peer-reviewed scholarly publications, unique historical collections from the University archives, and teaching tools. The Jefferson Digital Commons allows researchers and interested readers anywhere in the world to learn about and keep up to date with Jefferson scholarship. This article has been accepted for inclusion in CRMEHC Faculty Papers by an authorized administrator of the Jefferson Digital Commons. For more information, please contact: JeffersonDigitalCommons@jefferson.edu. 


\section{Authors}

Mohammadreza Hojat, Joseph S. Gonnella, Thomas J. Nasca, Sylvia K. Fields, Americo Cicchetti, Alessandra Lo Scalzo, Francesco Taroni, Anna Maria Vincenza Amicosante, Manuela Macinati, Massimo Tangucci, Carlo Liva, Gualtiero Ricciardi, Schmuel Eidelman, Hanna Admi, Hana Geva, Tanya Mashiach, Gideon Alroy, Adelina Alcorta-Gonzalez, David Ibarra, and Antonio Torres-Ruiz 


\title{
Comparisons of American, Israeli, Italian and Mexican Physicians and Nurses on the Total and Factor Scores of the Jefferson Scale of Attitudes toward Physician- Nurse Collaborative Relationships
}

\author{
Mohammadreza Hojat ${ }^{a}$ Joseph S. Gonnella ${ }^{a}$, Thomas J. Nasca ${ }^{a}$, Sylvia K. Fields ${ }^{a}$, \\ Americo Cicchetti ${ }^{b}$, Alessandra Lo Scalzo ${ }^{c}$, Francesco Taroni ${ }^{c}$, Anna Maria \\ Vincenza Amicosante ${ }^{c}$, Manuela Macinati ${ }^{b}$, Massimo Tangucci ${ }^{c}$, Carlo Liva ${ }^{c}$, \\ Gualtiero Ricciardi $^{\text {b }}$, Shmuel Eidelman ${ }^{d}$, Hanna Admi ${ }^{\text {, }}$, Hana Geva ${ }^{\text {, }}$, Tanya \\ Mashiach $^{d}$, Gideon Alroy ${ }^{d}$, Adelina Alcorta-Gonzalez ${ }^{\mathrm{e}}$, David Ibarra ${ }^{\mathrm{f}}$ and Antonio \\ Torres-Ruiz ${ }^{\mathrm{g}}$
}

${ }^{a}$ Center for Research in Medical Education and Health Care, Jefferson Medical College of Thomas Jefferson University, 1025 Walnut Street, Philadelphia, PA, 19107, USA

${ }^{\mathrm{b}}$ Health Economics Laboratory, Institute of Hygiene, Catholic University, Rome, Italy

${ }^{c}$ National Agency for Regional Health Services, Agenzia per i Servizi Saitari Regionali, ASSR, Rome, Italy

${ }^{d}$ Rambam Medical Center and Faculty of Medicine, Technion-Israel Institute of Technology, Haifa, Israel

${ }^{\mathrm{e}}$ School of Medicine, Autonomous University of Nuevo Leon, Monterrey, Mexico

${ }^{\mathrm{f}}$ Mexican Institute of Social Security, Mexico

${ }^{g}$ National Institute of Neurology and Neurosurgery, "Manuel Velasco Suarez", Mexico

Corresponding author: M. Hojat,

Email: mohammadreza.hojat@jefferson.edu

Tel.: +1-215-955-9495; Fax +1-215-923-6939

\begin{abstract}
This cross-cultural study was designed to compare the attitudes of physicians and nurses toward physician-nurse collaboration in the United States, Israel, Italy and Mexico. Total participants were 2522 physicians and nurses who completed the Jefferson Scale of Attitudes Toward Physician-Nurse Collaboration (15 Likert-type items, (Hojat et al., Evaluation and the Health Professions 22 (1999a) 208; Nursing Research 50 (2001) 123). They were compared on the total scores and four factors of the Jefferson Scale (shared education and team work, caring as opposed to curing, nurses, autonomy, physicians' dominance). Results showed inter- and intra-cultural similarities and differences among the study groups providing support for the social role theory (Hardy and Conway, Role Theory: Perspectives for Health Professionals, Appleton-Century-Crofts, New York, 1978 ) and the principle of least interest (Waller and Hill, The Family: A Dynamic Interpretation, Dryden, New York, 1951) in inter-professional relationships. Implications for promoting physician-nurse education and inter-professional collaboration are discussed.
\end{abstract}




\section{Introduction}

\subsection{Importance of physician-nurse collaboration in patient care}

Research generally suggests that interdisciplinary healthcare teamwork improves clinical outcomes as well as patient satisfaction, and can decrease institutional costs (Baggs et al., 1992; Cook, 1998; Feiger and Schmitt, 1979; Kosper et al., 1994; Rubenstein et al., 1984; Warner and Hutchinson, 1999). Correspondingly, collaborative relationships between nurses and physicians can positively influence clinical outcomes, such as patient dealth rates ( Knaus et al., 1986). Compelling evidence suggests that physician-nurse interprofessional collaboration can also help contain health care costs ( Blegen et al., 1995; Fagin, 1992; Gibson et al., 1994). In a recent study, a strong correlation was reported between nurses' perceptions of physician-nurse collaboration and satisfaction with decision making ( Dechario-Marino et al., 2001).

The link between the physician-nurse relationship and patient outcomes exists regardless of geographical boundaries. For example, a recent study in Sweden showed that positive physician-nurse relationships had a beneficial effect on the quality of drug use and the improvement of behavioral disturbances among a large number of nursing home residents (Schmidt and Svarstad, 2002).

Currently, the health care system is experiencing a shortage of nurses (Steinbrook, 2002). It is reported that this decline is partly due to unsatisfactory inter-professional relationships between physicians and nurses ( Blau and Ferber, 1992; Steinbrook, 2002). Due to the market demand for new nurses as well as the decline in interest to pursue nursing careers because of unsatisfactory physician-nurse relationships, there is a serious need to promote positive changes toward inter-professional collaboration between physicians and nurses ( Buerhaus et al., 2000; Rosenstein, 2002).

\subsection{Social and cultural factors in professional roles}

Physician-nurse collaboration is described as "nurses and physicians cooperatively working together, sharing responsibilities for solving problems and making decisions to formulate and carry out plans for patient care." (Baggs and Schmitt, 1988, p. 145). The extent of mutual collaboration between physicians and nurses can be influenced not only by educational factors, but also by prescribed societal roles and cultural norms ( Fagin, 1992; Hardy and Conway, 1978; Makaram, 1995). Although the influence of social and cultural factors was originally addressed by social psychologists (e.g., Sherif et al., 1965), researchers have specifically discussed the influence of attitudes formed by cultural norms and role socialization in interpersonal relationships among health care workers ( Conway, 1978). Educational remedies to promote positive changes in inter-professional collaboration within the framework of the social role theory have also been discussed ( Conway, 1978).

According to the tenet of the social role theory, attitudes and expectations in different cultures play key roles in physician-nurse collaborative relationships (Meleis and 
Hassan, 1980; Ornstein, 1990). For example, it is reported that the relationship between physicians and nurses tends to be "hierarchical" in societies where nurses have very little autonomy, while physicians have a total dominance in patient care decisions (Austin et al., 1985; Champion et al., 1987; Meleis and Hassan, 1980). In contrast, interprofessional relationships tend to be "complementary" in societies where physicians and nurses share the power and are viewed to have complementary roles and responsibilities in patient care.

The hierarchical model places more emphasis on factors such as the gender divisions of labor in society (Sweet and Norman, 1995), professional elitism, and gender role stereotypes ( Blickensderfer, 1996; Fagin, 1992; Giardino et al., 1994; Hojat et al., 1997; McMahan et al., 1994; Prescott and Bowen, 1985; Sprague-McRae, 1996). This model naturally places medicine far above nursing in patient care responsibilities ( Shein, 1972). As a result, adversarial relationships can develop between physicians and nurses ( Blickensderfer, 1996; Huntington and Shores, 1993; McMahan et al., 1994; PavlovichDanis et al., 1998; Prescott and Bowen, 1985).

The complementary model places more emphasis on the importance of education, common experiences, shared autonomy, and mutual authority. Attitudes toward interprofessional collaboration are expected to be more positive in societies in which a complementary model is promoted in the formal education of health professionals.

Research suggests that social stereotyping and cultural norms can strongly influence different aspects of the complex dynamics of the physician-nurse relationships (Austin et al., 1985; Champion et al., 1987; Davidhizar et al., 1990; Leininger, 1978; Ornstein, 1990). For example, in Middle East cultures, nurses are more often perceived as physicians' "handmaidens." In these cultures the hierarchical model of professional roles is more prevalent (Meleis and Hassan, 1980).

The cultural views on nurses' autonomy and their subordination in Italian (Sala and Manara, 1999; Sala and Usai, 1997) and Mexican ( Warda, 2000) cultures suggest that professional roles of physicians and nurses lean more toward hierarchical than complementary models. Israeli medical and nursing education is modeled after the United States' with similar contents in health care issues ( Bergman et al., 1975; Ehrenfeld et al., 1992; Ehrenfeld and Eckerling, 1995; Kater, 2000; Yagil et al., 2001). A complementary model of physician-nurse relationship is encouraged in medical and nursing education in these countries.

\subsection{Greater professional power and less desire for collaboration}

It has been proposed that those in a greater power position are less likely to express a desire for a collaborative relationship. This notion was first described as the "principle of least interest" by Waller and Hill (1951) in the context of family relationships. Based on the principle of least interest, we can expect physicians to express less orientation toward sharing powers and a full collaboration with nurses since they have traditionally been in a more of a power position. 
The unprecedented changes that are occurring in the health care systems at national and international levels can change the expectations. For example, teamwork is increasing viewed as necessary to contain costs and sustain the quality of care. These factors influence the expectations of physicians and nurses about their professional collaboration, but market demands and corporate medicine in different cultures may influence collaborative relationships between physicians and nurses differently.

The transitional changes in the health care system require adjustments in educational curriculum and in the professional roles of physicians and nurses in different countries with different cultural norms. Therefore, it is important and timely to examine intercultural and intra-cultural similarities and differences in attitudes toward physician-nurse relationships to better understand their contribution to professional roles and expectations. Such understanding can help to enhance inter-professional education in different cultures for the purpose of increasing professional satisfaction and for improving patient outcomes, regardless of geographical boundaries.

\subsection{Multi-dimensional and multi-cultural nature of physician-nurse relationships}

Despite its importance, empirical research on physician-nurse collaborative relationships in different cultures has not received sufficient attention. This is partly due to the unavailability of a multi-dimensional and psychometrically sound research instrument that is applicable to both physicians and nurses in different cultures. A brief instrument (6 items) has been developed by Baggs (1994) for measuring physician-nurse collaboration on patient care decisions, but it is a uni-dimensional tool that cannot address the multifaceted nature of physician-nurse collaboration.

In response to a need for a multi-dimensional tool for measuring different aspects of physician-nurses collaborative relationships, we developed the Jefferson Scale of Attitudes Toward Physician-Nurse Collaboration (Hojat and Herman, 1985; Hojat et al. (1997) and Hojat et al. (1999a)). We will describe this scale in more detail later. In a recent study in which we used this scale with American and Mexican physicians and nurses, we noticed interesting differences in the expected directions on the total scores of the scale ( Hojat et al., 2001). The current study expands on our previous research by including data for physicians and nurses from four countries with different social and cultural role expectations, and by examining scores on different factors (aspects) of attitudes toward physician-nurse collaboration in these countries.

\subsection{Research purposes}

Based on the notion that attitudes toward different aspects of physician-nurse collaboration could reflect the predominant cultural model of professional roles, we expected to find more similarities in attitudes toward physician-nurse collaboration between American and Israeli samples than between these groups and their Italian and

Mexican counterparts. This expectation was based on the presumption that in the United States and Israel the roles of physicians and nurses are likely to be viewed from a 
complementary model perspective. This is in contrast to their Italian and Mexican counterparts who presumably see a more hierarchical structure of professional roles.

Furthermore, we expected to find that nurses would express more positive attitudes than physicians toward collaborative relationships in all four countries. This expectation was based on the principle of least interest proposed by Waller and Hill (1951) that was described previously.

\section{Method}

\subsection{Participants}

Total participants were 2522 physicians and nurses in the United States $(n=202,118$ physicians, 84 nurses), Israel ( $n=602,156$ physicians and 446 nurses), Italy ( $n=1287$, 428 physicians and 859 nurses), and Mexico ( $n=435,148$ physicians, and 287 nurses). A convenience sampling method was used, and participating was voluntary. Of the total physicians in the United States, $35 \%(n=42)$ were women. There were $28 \%$ women in the Israeli sample of physicians $(n=43)$. Among Italians physicians $22 \%(n=89$ of 396 who specified their sex) were women, and of the total Mexican physicians $26 \%(n=39)$ were women. Of the total American nurses, $86 \%(n=72)$ were women; of Israeli nurses, $87 \%(n=386)$ were women; of the total Italian nurses who specified their sex $(n=773)$, $73 \%(n=564)$ were women; and 91\% $(n=261)$ of Mexican nurses were women. All nurses in the study were hospital-based and involved in direct patient care. Physicians' self-reported specialties for American and Mexican samples included internal medicine and medical sub-specialties, surgery and surgical sub-specialties and psychiatry. Specialty information was available for about half of the Israeli physicians who were based in a secondary and tertiary care hospitals. Specialty areas of Italian physicians were internal medicine and medical subspecialties, surgery and subspecialties, and pediatrics.

\subsection{Instrument}

The Jefferson Scale of Attitudes toward Physician-Nurse Collaboration was used in this study. The scale was originally developed to measure attitudes toward nurses and nursing services (Hojat and Herman, 1985). Items for the original scale were selected based on a review of literature on physician-nurse relationships and addressed areas of physiciannurse interactions, decision making, role expectations, authority, autonomy, and responsibilities for patient care and monitoring. That original survey was modified ( Hojat et al., 1997) to investigate attitudes toward physician-nurse alliances. Further modifications were made to the survey in a third study (Hojat et al., 1999a) in which 15 (out of the 20) items of the survey were retained after extensive psychometric analysis.

The items in the final version of the Jefferson Scale of Attitudes toward Physician-Nurse Collaboration are answered on a 4-point Likert-type scale from "strongly agree" to "strongly disagree." A higher total score reflects a more positive attitude toward physician-nurse collaborative relationships. Findings of our previous study showed that nursing students obtained a significantly higher average score on the total scale than 
medical students (Hojat et al., 1999a). Reliability coefficients for this scale for medical and nursing students were in the $0.80 \mathrm{~s}$ (Hojat et al., 1999a).

Results of an exploratory factor analysis provided support for the construct validity of this research tool. The prominent underlying factors of the Jefferson Scale of Attitudes toward Physician-Nurse Collaboration (Hojat et al., 1999a) were identified as "shared education and team work" (7 items, a typical item: "During their education, medical and nursing students should be involved in teamwork in order to understand their respective roles.") Three other reliable factors were "caring as opposed to curing" (three items, a typical item: "Nurses are qualified to assess and respond to psychological aspects of patients' needs."); "nurse's autonomy" (three items, a typical item: "Nurses should be involved in making policy decisions concerning the hospital support services on which their work depends."); and "physician's dominance" (two items, a typical item: "The primary function of the nurse is to carry out the physician's orders.")

A higher factor score on the shared education and teamwork dimension indicates a greater orientation toward interdisciplinary education and inter-professional collaborations. A higher factor score on the caring, as opposed to curing, dimension indicates a more positive view of nurses' contributions to psychosocial and educational aspects of patient care. A higher factor score on the nurses' autonomy dimension indicates more agreement with nurses' involvement in decisions on patient care and policies. A higher factor score on physicians' dominance indicates rejecting a totally dominant role of physicians in aspects of patient care (items of this factor are reverse scored).

\subsection{Procedures}

The Jefferson Scale of Attitudes toward Physician-Nurse Collaboration was translated from English into Hebrew (by one of the authors, Shmuel Eidelman and his research team in Israel), into Italian (by Americo Cicchetti and Alessandra Lo Scalzo, Manuela Macinati and Lamberto Manzoli), and into Spanish by two of the co-authors in Mexico (Adelina Alcorta-Gonzalez and David Ibarra). The Hebrew, Italian and Spanish versions were then back-translated into English by bilingual researchers to ensure the accuracy of the translation (Brislin, 1970).

The English version of the instrument was distributed to physicians and nurses at Thomas Jefferson University Hospital in Philadelphia in the United States. The Hebrew version was distributed in Haifa, Israel to physicians and nurses affiliated with Rambam Medical Center and the Technion Faculty of Medicine. The Italian version was distributed to physicians and nurses in one university-affiliated hospital (A. Gemelli Hospital) and two public hospitals (S. Camillo, and Forlanini) located in Roma. The Spanish version of the scale was distributed to physicians and nurses in a university-affiliated and a public hospital in Mexico. The university-affiliated hospital was the University Hospital of the "Jose E. Gonzalez" University Hospital of School of Medicine at the Autonomous University of Nuevo Leon (UANL) in Monterrey, and the public hospital was Hospital Angeles del Pedregal, de las Lomas of Mexican Institute of Social Security. The survey

Author's final version prior to publication. See International Journal of Nursing Studies 40(4):427-435, May 2003, for the copy of record. 
was anonymous. Respondents did not sign their names, but they were asked to voluntarily provide information on their age, gender, professional status, and areas of specialization.

\subsection{Statistical analyses}

We transformed the scores for the four factors to a standard distribution with a mean of 100 and a standard deviation of 10 for easier and more meaningful comparisons on a unique scale for all four factors. Comparisons of the total scores of the Jefferson Scale of Attitudes toward Physician-Nurse Collaboration were made on the raw scores. We employed a two-way multivariate analysis of variance (MANOVA) to simultaneously compare the scores on the four factors (dependent variables) by two independent variables: Country (US, Israel, Italy and Mexico) and profession (medicine, nursing).

The multivariate $F$-ratios for the main effects of country and profession, as well as their interactions, were statistically significant. Because of the significant interaction effects, we decided to use one-way MANOVA in which the eight groups (American nurses, American physicians, Israeli nurses, Israeli physicians, Italian nurses, Italian physicians, Mexican nurses, and Mexican physicians) were treated as the levels of the independent variable. Post hoc mean comparisons were made simultaneously on the means for the four factors in the eight study groups. Similar analyses were performed for the total scale scores by using univariate analysis of variance (ANOVA). Analysis of covariance (ANCOVA) was also used to control for gender and age.

\section{Results}

Prior to group comparisons, we examined the scale's internal consistency aspect of reliability by calculating coefficient alpha for the eight study groups. Results are reported in Table 1 . The reliability coefficients ranged from 0.70 (for Israeli and Italian nurses) to 0.86 (for Mexican physicians). Reliability coefficients of these magnitudes are in the acceptable range for attitude scales.

Table 1.

Reliability coefficients ${ }^{\mathrm{a}}$ of the Jefferson scale of attitudes toward physician-nurse collaboration for $\mathbf{2 5 2 6}$ American, Israeli, Italian, and Mexican physicians and nurses.

\begin{tabular}{lcc} 
Country & Physicians & Nurses \\
\hline United States & & \\
Israel & 0.78 & 0.74 \\
Italy & 0.78 & 0.70 \\
Mexico & 0.76 & 0.70 \\
& 0.86 & 0.79
\end{tabular}

\footnotetext{
${ }^{\mathrm{a} C o e f f i c i e n t ~ a l p h a . ~}$
} 
Prior to group comparisons, we examined the total scores of the attitude scale between Mexican samples from the university-affiliated and public hospitals. No substantial difference was observed between the two samples (physicians and nurses were compared separately); therefore, data for participants from the two Mexican hospitals were combined for statistical analyses. Also, since no substantial statistically significant differences were observed among Italians in the three different hospitals, data for the three hospitals were combined for cross-cultural comparisons.

Table 2 shows the means and standard deviations of the total scale scores and of the four factors, the number of observations in each study group, and summary results of statistical analyses.

Table 2. Comparisons of American, Israeli, Italian and Mexican physicians and nurses on total scores and four factors of the Jefferson scale of attitudes toward physician-nurse collaborationa

Factors

\begin{tabular}{|c|c|c|c|c|}
\hline $\begin{array}{l}\text { Shared education } \\
\text { and team work } \\
(M \pm S D)\end{array}$ & $\begin{array}{l}\text { Caring vs. curing } \\
(M \pm S D)\end{array}$ & $\begin{array}{l}\text { Nurses' } \\
\text { autonomy } \\
(M \pm S D)\end{array}$ & $\begin{array}{l}\text { Physicians' } \\
\text { dominance } \\
(M \pm S D)\end{array}$ & $\begin{array}{l}\text { Total } \\
\text { score } \\
(M \pm S D)\end{array}$ \\
\hline
\end{tabular}

\begin{tabular}{|c|c|c|c|c|c|}
\hline 1. Physicians $(n=118)$ & $97 \pm 9.5$ & $99 \pm 8.5$ & $99 \pm 8.2$ & $99 \pm 7.7$ & $48 \pm 4.9$ \\
\hline 2. Nurses $(n=84)$ & $107 \pm 5.9$ & $105 \pm 6.6$ & $105 \pm 6.5$ & $109 \pm 7.1$ & $55 \pm 3.6$ \\
\hline 3. Physicians $(n=156)$ & $102 \pm 9.5$ & $98 \pm 11.5$ & $101 \pm 9.9$ & $91 \pm 8.0$ & $49 \pm 5.3$ \\
\hline 4. Nurses $(n=146)$ & $107 \pm 5.9$ & $104 \pm 7.2$ & $103 \pm 6.6$ & $102 \pm 9.1$ & $54 \pm 3.8$ \\
\hline 5. Physicians $(n=428)$ & $94 \pm 11.0$ & $95 \pm 11.0$ & $99 \pm 9.7$ & $92 \pm 8.7$ & $45 \pm 5.8$ \\
\hline 6. Nurses $(n=859)$ & $100 \pm 9.1$ & $103 \pm 7.9$ & $102 \pm 8.6$ & $103 \pm 8.8$ & $51 \pm 4.7$ \\
\hline 7. Physicians $(n=148)$ & $97 \pm 9.2$ & $91 \pm 11.5$ & $88 \pm 10.4$ & $96 \pm 7.4$ & $45 \pm 6.3$ \\
\hline 8. Nurses $(n=287)$ & $99 \pm 9.8$ & $97 \pm 9.3$ & $93 \pm 11.7$ & $102 \pm 9.6$ & $48 \pm 5.5$ \\
\hline Univariate $F_{(5,1226)}$ & $81.1 * *$ & $82.8 * *$ & $84.6^{* *}$ & $113.6^{* *}$ & $132.7 * *$ \\
\hline Group differences & $\begin{array}{l}2=4>3> \\
1=6=7=8>5\end{array}$ & $\begin{array}{l}2=4=6> \\
1=3=8>5>7\end{array}$ & $\begin{array}{l}2=4=6> \\
1=3=5>8>7\end{array}$ & $\begin{array}{l}2>4=6=8> \\
1>7>3=5\end{array}$ & $\begin{array}{l}2>4>6> \\
1=3=8>5=7\end{array}$ \\
\hline
\end{tabular}

\footnotetext{
${ }^{* * *} P<0.01$.

${ }^{a}$ Factor scores for the entire samples were transformed to a standard distribution with a mean of 100 and a standard deviation of 10. Wilkes' lambda $=0.48$, multivariate $F_{(28,9040)}=73.7 * *$ (for the 4 factors).
}

\subsection{Comparisons of total scores}

Author's final version prior to publication. See International Journal of Nursing Studies 40(4):427-435, May 2003, for the copy of record. 
The results of ANOVA followed by Duncan post hoc mean comparison test showed significant differences among groups $\left(F_{(7,2514)}=132.7, p<0.01\right)$. As reported in the table, the highest mean score was obtained by the American nurses followed by Israeli nurses, and the lowest mean score was obtained by Mexican and Italian physicians. The mean scores obtained by the American physicians, Israeli physicians and Mexican nurses were not significantly different, but were significantly lower than those obtained by the American, Israeli and Italian nurses, and significantly higher than those obtained by Italian and Mexican Physicians. These findings indicate that the American, Israeli and Italian nurses expressed the most positive attitudes toward physician-nurse collaboration, and Italian and Mexican physicians expressed the least positive attitudes.

Regardless of the country, nurses scored $(M=51.5)$ significantly higher than physicians $(M=46.3)$ on the total attitude scale, $F_{(1,2520)}=541.4, p<0.01$. Regardless of professions, Israelis obtained the highest mean score $(M=52.3)$, followed by Americans $(M=51.1)$, Italians $(M=49.2)$, and Mexicans $(M=47.1)$. All these cross-cultural differences on the total scores were statistically significant: $F_{(3,2518)}=80.6, p<0.01$.

\subsection{Comparisons on factor scores}

As reported in Table 2, the results of multivariate analysis indicated significant differences among the groups on factor scores (lambda $=0.48$, multivariate $F_{(28,9040)}=$ 73.6, $p<0.01)$. A general pattern was observed in which the highest mean scores on three factors of "shared education and team work," "caring vs. curing," and "nurses' autonomy" were obtained by American and Israeli nurses, which was significantly different from any other physician group.

On the factor of "shared education and team work" the Italian physicians obtained the lowest mean score, followed by American physicians, Mexican physicians and nurses, and Italian nurses. The Israeli physicians scored significantly higher than all of the aforementioned groups, but significantly lower than American and Israeli nurses.

On the factor of "caring vs. curing" the Italian physicians scored lower than any other group, and American, Italian and Israeli nurses scored significantly higher than all other groups. On the factor of "nurses' autonomy," the American, Italian, and Israeli nurses obtained the highest mean scores, and Mexican physicians and nurses obtained the lowest mean scores. On the factor of "physician's dominance," the American nurses obtained the highest mean scores, followed by nurses in the three countries, and the lowest mean scores were obtained by Israeli and Italian physicians, which was significantly different from other groups.

Additional analyses were performed in which gender and age were controlled by analysis of covariance (gender and age as covariates). The general patterns of the aforementioned findings remained unchanged indicating that the obtained results were not confounded by these demographic variables.

\section{Discussion}

Author's final version prior to publication. See International Journal of Nursing Studies 40(4):427-435, May 2003, for the copy of record. 
Findings of this study generally confirmed our expectations. The intercultural comparisons indicated that, regardless of the profession (physicians and nurses combined), the Italian and Mexican samples obtained the lowest mean attitude scores on the total, as well as on the two factors of shared education and team work and caring vs. curing. On the factor of nurses' autonomy, Israelis had the highest and Mexicans had the lowest mean scores, which was significantly different than their American and Italian counterparts.

Nurses from countries in which the complementary model of professional roles is prevalent (United States and Israel) expressed more positive attitudes toward physiciannurse collaboration than their Italian and Mexican counterparts where a hierarchical model of professional roles is more common. These findings provided evidence in support of the socialization role theory (Austin et al., 1985; Champion et al., 1987; Conway, 1978; Hardy and Conway, 1978; Meleis and Hassan, 1980; Ornstein, 1990).

Intra-cultural comparisons showed attitudinal discrepancies between physicians and nurses within each country. Our findings showed that nurses desire collaborative physician-nurse relationship more than physicians, regardless of cultural differences. This is consistent with recent findings on attitudes toward physician-nurse relationships in which physicians viewed the relationship as less important than did nurses (Rosenstein, 2002). These findings are also consistent with the prediction based on the Waller and Hall's (1951) principle of least interest which was described previously.

We calculated the effect size estimates of the mean differences to investigate the practical significance of the statistically significant differences found between physicians and nurses. On the total scores of the attitude scale, the largest discrepancy was found between physicians and nurses in the United States (effect SIZE $=1.3$ ), followed by Israelis (effect SIZE $=1.1$ ) and Italians (effect SIZE $=0.90)$. The lowest effect size was found between Mexican physicians and nurses (effect SIZE $=0.50$ ). According to the operational definitions of effect size estimates proposed by Cohen (1987, p. 40), effect sizes around 0.25 are small and negligible, those around 0.50 are moderate, and above 0.80 are large and important. By these definitions, the discrepancies between American, Israeli, and Italian physicians and nurses are practically more important than those between the Mexican groups. These findings indicate that the gap between physicians and nurses varies among countries with different health care systems ( Aiken et al., 2001), and unexpectedly, the gap is wider in countries in which with a complementary professional role is apparently encouraged.

Intra-cultural comparisons on the four factors associated with physician-nurse collaboration showed that the largest gaps were between American physicians and nurses, and between Israeli physicians and nurses on the factor of "physicians' dominance" (effect SIZE = 1.1). The lowest attitudinal discrepancies were observed between Mexican physicians and nurses on the factor of "shared education and team work" (effect SIZE = $0.20)$ and between Italian physicians and nurses on the factor of "nurses' autonomy" (effect SIZE $=0.30$ ). The discrepancies between physicians and nurses among Mexican and Italian samples were mostly moderate based on the calculated effect size estimates.

Author's final version prior to publication. See International Journal of Nursing Studies 40(4):427-435, May 2003, for the copy of record. 
The pattern of intra-cultural comparison findings generally suggests that attitudes of physicians and nurses toward inter-professional collaboration in Italy and Mexico are more congruent than those in the US and Israel. These findings suggest that less conflict in the professional roles of physicians and nurses in countries with more traditional professional roles (Mexico and Italy) may be expected. On the contrary, there is a deeper gap between physicians and nurses in societies where a complementary role model is encouraged (US and Israel). Most likely, role ambiguity is the cause of the discrepancy (Weiss, 1983). This pattern of unexpected findings is important and deserves further empirical scrutiny in future studies.

The patterns of findings in the four countries were independent of gender and age. This suggests that those demographic variables are not as powerful as social learning and cultural factors in forming attitudes toward physician-nurse relationships (Hardy and Conway, 1978).

\subsection{Study limitations}

The internal validity of the findings can be improved by collecting more data on the psychometric properties of the Jefferson Scale of Attitudes toward Physician-Nurse Collaboration in other cultures. Although the face validity of the items of the Scale was confirmed in the process of translation and back-translation into Italian, Hebrew and Spanish languages, more validity evidence is needed. The coefficient alpha, an indicator of the internal consistency aspect of reliability was found satisfactory in all of the translated versions, but we need more data on score stability over-time (test-retest reliability).

The convenience sampling used in the present study may limit the generalization of the findings to a broader population of physicians and nurses. Replication of the study can provide assurances for the external validity (generalizability) of the findings.

\section{Conclusions}

Collaborative relationships between physicians and nurses in any culture benefit patients and contribute to better communication and satisfaction within the professions (Baggs et al., 1997; Rosenstein, 2002). As we described before ( Hojat et al., 2001), it is desirable that medical and nursing schools in different countries include inter-professional education in their curriculum to promote shared experiences and a better understanding of the roles of physicians and nurses. Such understanding will narrow the gap between physicians and nurses and foster mutually respectful inter-professional relationships between the two professions ( Szasz, 1969; Wieczorek et al., 1976). Shared education and common experiences can modify socially prescribed stereotypical roles and can contribute to a better acceptance of a complementary model of professional roles.

The study of physician-nurse collaborative relationships and the implementation of educational programs to improve such relationships are particularly important considering the forecasted shortage of nurses and the decreased interest in the nursing 
profession, particularly in the United States (Buerhaus et al., 2000; Rosenstein, 2002). In addition, the unprecedented and inevitable changes that are universally taking place in the health care delivery systems call for innovative approaches to improve physician-nurse collaboration in order to contain cost and maintain the quality of care. A growing concern exists worldwide about cost containment and quality of care (Gonnella and Hojat, 2001; Hojat et al., 1999b). Teamwork and inter-professional collaboration between physicians and nurses can curtail costs and improve the quality of care and team effectiveness ( Dechario-Marino, et al., 2001; Hojat et al., 2000; Pavlovich-Danis et al., 1998; Stichler, 1995; Walton, 1995).

Because physician-nurse collaboration can improve the level of care given to the patient, it is desirable to examine different factors of inter-professional collaborations not only within a given culture but also between different cultures. The multi-dimensional research tool used in this study can serve as an operational measure to assess interdisciplinary educational programs and to evaluate group changes, similarities, and differences within and between cultures.

\section{Acknowledgements}

We would like to thank Guadalupe Ibarra and Alma Garcia for their contribution in collecting data from Mexican physicians and nurses, Lamberto Manzoli for his contribution in translating the scale into Italian, Laura Pellegrini, Director of the National Agency for Regional Health Services in Italy, Professor Gianfranco Vanini, Director of the Catholic University Hygiene Institute in Italy, Guido Pugliesi, Director General of S. Camillo Forlanini Hospital Trust in Italy, Antonio Cicchetti, Director General of A. Gemelli Hospital in Italy, Giacomo Furnari, Daniele Palumbo, Caterina Amoddeo, Maria Rosaria Cocco, Roberta Vuchelich, Sara Catania, Ilaria Misticoni Consorti, Ugo Monticone, Ciro Vinciguerra, Claudia Fariello, and Dario Fella for their help on the project in Italy, and Dorissa Bolinski for her editorial assistance.

\section{References}

Aiken, L.H., Clarke, S.P., Sloane, D.M., Sochalski, J.A., Busse, R., Clarke, H., Giovannetti, P., Hunt, J., Rafferty, A.M. and Shamian, J., 2001. Nurses' report on hospital care in five countries. Health Affairs 20, pp. 43-53.

Austin, J., Champion, V. and Tzeng, O.C.S., 1985. Cross-cultural comparison on nursing image. International Journal of Nursing Studies 22, pp. 231-239.

Baggs, J.G., 1994. Development of an instrument to measure collaboration and satisfaction about care decisions. Journal of Advanced Nursing 20, pp. 176-182.

Baggs, J.G. and Schmitt, M.H., 1988. Collaboration between nurses and physicians. Image: Journal of Nursing Scholarship 20, pp. 145-149. 
Baggs, J.G., Ryan, S., Phelps, C.E., Richardson, J.F. and Johnson, J.E., 1992. The association between interdisciplinary collaboration and patient outcomes in a medical intensive care unit. Heart and Lung 21, pp. 18-24.

Baggs, J.G., Schmitt, M.H., Mushlin, A.I., Eldregde, D.H., Oakes, D. and Hutson, A.D., 1997. Nurse-physician collaboration and satisfaction with the decision-making process in three critical care units. American Journal of Critical Care 6, pp. 393-399.

Bergman, R., Shavitt, N., Ditzian, I. and Malamed, Y., 1975. Work-life of the Israeli registered nurse. International Journal of Nursing Studies 12, pp. 133-168.

Blau, F.D. and Ferber, M.A., 1992. The Economics of Women, Men and Work (2nd Edition ed.),, Prentice-Hall, Englewood Cliffs, NJ.

Blegen, M.A., Reiter, R.C., Goode, C.J. and Murphy, R.R., 1995. Outcomes of hospitalbased managed care: a multivariate analysis of cost and quality. Obstetrics and Gynecology 86, pp. 809-814.

Blickensderfer, L., 1996. Nurses and physicians: creating a collaborative environment. Journal of Intravenous Nursing 19, pp. 127-131.

Brislin, R.W., 1970. Back translation for cross cultural research. Journal of CrossCultural Psychology 1, pp. 185-216.

Buerhaus, P.I., Staiger, D.O. and Auerbach, D.I., 2000. Implications of an aging registered nurse workforce. Journal of American Medical Association 283, pp. 2848 2954.

Champion, V., Austin, J. and Tzeng, O.C.S., 1987. Cross-cultural comparison of images of nurses and physicians. International Nursing Review 34, pp. 43-48.

Cohen, J., 1987. Statistical Power Analysis for Behavioral Sciences, Erlbaum, Hillsdale.

Conway, M.E., 1978. Theoretical approaches to the study of roles. In: Hardy, M.E. and Conway, M.E., Editors, 1978. Role theory: Perspectives for Health Professionals, Appleton-Century-Crofts, New York, pp. 17-27.

Cook, T.H., 1998. The effectiveness of inpatient case management: fact or fiction?. Journal of Nursing Administration 28, pp. 36-46.

Davidhizar, R., Policinski, H. and Bowen, M., 1990. The difficult doctor. Today's OR Nurse 12, pp. 28-30.

Dechario-Marino, A.E., Jordan-Marsh, M., Traiger, G. and Saulo, M., 2001. Nurse/physician collaboration: action research and the lessons learned. Journal of Nursing Administration 31, pp. 223-232. 
Ehrenfeld, M. and Eckerling, S., 1995. Professional expectations and activities of nurses in Israel. Journal of Nursing Management 3, pp. 177-182.

Ehrenfeld, M., Bergman, R. and Ziv, L., 1992. Academia: a stimulus for change.

International Nursing Review 39, pp. 23-26.

Fagin, C.M., 1992. Collaboration between nurses and physicians: no longer a choice. Academic Medicine 67, pp. 295-303.

Feiger, S.M. and Schmitt, M.H., 1979. Collegiality in interdisciplinary health teams: its measurement and its effects. Social Science \& Medicine 13, pp. 217-229.

Giardino, A.P., Giardino, E.R. and Siegler, E.L., 1994. Teaching collaboration to nursing and undergraduates. In: Siegler, E.L. and Whitney, F.W., Editors, 1994. Nurse-physician Collaboration: Care of Adults and Elderly, Springer-Verlag, New York, pp. 127-157.

Gibson, S.J., Martin, S.M., Johnson, M.B., Blue, R. and Miller, D.S., 1994. CNS directed case management: Cost and quality in harmony. Journal of Nursing Administration 24, pp. $45-51$.

Gonnella, J.S. and Hojat, M., 2001. Biotechnology and ethics in medical education of the new millennium: physician roles and responsibilities. Medical Teacher 23, pp. 371-377.

Hardy, M.E., Conway, M.E. (Eds.). 1978. Role Theory: Perspectives for Health Professionals. Appleton-Century-Crofts, New York.

Hojat, M. and Herman, M.W., 1985. Developing and instrument to measure attitudes toward nurses: preliminary psychometric findings. Psychological Reports 56, pp. 571579.

Hojat, M., Fields, S.K, Rattner, S.L., Griffiths, M., Cohen, M.J.M. and Plumb, J.D., 1997. Attitudes toward physician-nurse alliance: comparisons of medical and nursing students. Academic Medicine 72, pp. S1-S3.

Hojat, M., Fields, S.K., Veloski, J.J., Griffiths, M., Cohen, M.J.M. and Plumb, J.D., 1999. Psychometric properties of an attitude scale measuring physician-nurse collaboration. Evaluation and the Health Professions 22, pp. 208-220.

Hojat, M., Veloski, J.J., Louis, D.Z., Xu, G., Ibarra, D., Gottlieb, J.E. and Erdmann, J.B., 1999. Perception of medical school seniors of the current changes in the US health care system. Evaluation and the Health Professions 22, pp. 169-183.

Hojat, M., Gonnella, J.S., Erdmann, J.B., Veloski, J.J., Louis, D.Z., Nasca, T.J. and Rattner, S.L., 2000. Physicians' perceptions of the changing health care system: comparisons by gender and specialties. Journal of Community Health 25, pp. 455-471.

Author's final version prior to publication. See International Journal of Nursing Studies 40(4):427-435, May 2003, for the copy of record. 
Hojat, M., Nasca, T.J., Cohen, M.J.M., Fields, S.K., Rattner, S.L., Griffiths, M., Ibarra, D., Alcorta-G de Gonzalez, A., Torres-Ruiz, A., Ibarra, G. and Garcia, A., 2001.

Attitudes toward physician-nurse collaboration: a cross-cultural study of male and female physicians and nurses in the United States and Mexico. Nursing Research 50, pp. 123128.

Huntington, J.A. and Shores, L., 1993. From conflict to collaboration. American Journal of Nursing 83, pp. 1184-1186.

Kater, V., 2000. A tale of teaching two cities. International Nursing Review 47, pp. 121125.

Knaus, W.a., Draper, E.A., Wagner, D.P. and Zimmerman, J.E., 1986. An evaluation of outcome from intensive care in major medical centers. Annals of Internal Medicine 104, pp. 410-418.

Kosper, K.G., Horn, P.B. and Carpenter, A.D., 1994. Successful collaboration within an integrative practice model. Clinical Nurse Specialist 8, pp. 330-333.

Leininger, M., 1978. Professional, political, and ethnocentric role bahaviors and their influence in multidisciplinary health education. In: Hardy, M.E. and Conway, M.E., Editors, 1978. Role Theory: Perspectives for Health Professionals, Appleton-CenturyCrofts, New York, pp. 251-271.

Makaram, S., 1995. Interprofessional cooperation. Medical Education 29, pp. 65-69.

McMahan, E., Hoffman, K. and McGee, G., 1994. Physician-nurse relationships in clinical settings: a review and critique of the literature, 1966-1992. Medical Care Review 51, pp. 83-112.

Meleis, A.I. and Hassan, H.S., 1980. Oil rich, nurse poor: the nursing crisis in the Persian gulf. Nursing Outlook 28, pp. 238-243.

Ornstein, H.J., 1990. Collaborative practice between Ontario nurses and physicians: is it possible?. Canadian Journal of Nursing Administration 3, pp. 10-14.

Pavlovich-Danis, S., Forman, H. and Simek, P.P., 1998. The nurse-physician relationship: can it be saved?. Journal of Nursing Administration 28, pp. 17-20.

Prescott, P.A. and Bowen, S.A., 1985. Physician-nurse relationships. Annals of Internal Medicine 103, pp. 127-133.

Rosenstein, A.H., 2002. Nurse-physician relationship: impact on nurse satisfaction and retention. American Journal of Nursing 102, pp. 26-34. 
Rubenstein, L.Z., Josephson, K.R., Weiland, G.D., English, P.A., Sayer, J.A. and Kane, R.L., 1984. Effectiveness of geriatric evaluation unit. The New England Journal of Medicine 311, pp. 1664-1670.

Sala, R. and Manara, D., 1999. The regulation of autonomy in nursing: the Italian situation. Nursing Ethics: An International Journal for Health Care Professionals 6, pp. $451-467$.

Sala, R. and Usai, M., 1997. Industrial action by nurses: the Italian situation. Nursing Ethics: An International Journal for Health Care Professionals 4, pp. 330-338.

Schmidt, I.K. and Svarstad, B.L., 2002. Nurse-physician communication and quality of drug use in Swedish nursing homes. Social Science \& Medicine 54, pp. 1767-1777.

Shein, E., 1972. Professional Education, McGraw-Hill, New York.

Sherif, C.W., Sherif, M. and Nebergall, R.E., 1965. Attitudes and Attitude Change: the Social Judgement-Involvement Approach, Saunders, Philadelphia.

Sprague-McRae, J.M., 1996. The advanced practice nurse and physician relationship: considerations for practice. Advanced Practice Nursing Quarterly 2, pp. 33-40.

Steinbrook, R., 2002. Nursing in the crossfire. The New England Journal of Medicine 346, pp. 1757-1766.

Stichler, J.F., 1995. Professional interdependence: the art of collaboration. Advanced Practice Nursing Quarterly 1, pp. 53-61.

Sweet, S.J. and Norman, I., 1995. The nurse-physician relationship: a selective literature review. Journal of Advanced Nursing 22, pp. 165-170.

Szasz, G., 1969. Interprofessional education in the health sciences. Millbank Memorial Fund Quarterly 47, pp. 449-475.

Waller, W. and Hill, R., 1951. The Family: A Dynamic Interpretation, Dryden, New York.

Walton, J.B., 1995. Multidisciplinary education. Medical Education 29, pp. 329-331.

Warda, M.R., 2000. Mexican-Americans' perceptions of culturally competence care. Western Journal of Nursing Research 22, pp. 203-204.

Warner, P.M. and Hutchinson, C., 1999. Heart failure management. Journal of Nursing Administration 29, pp. 28-37. 
Weiss, S.J., 1983. Role differentiation between nurse and physician: implications for nursing. Nursing Research 32, pp. 133-139.

Wieczorek, R., Pennington, E. and Fields, S., 1976. Interdisciplinary education: a model for the resocialization of faculty. Nursing Forum 15, pp. 224-237.

Yagil, D., Spitzer, A. and Ben-Zer, H., 2001. From bedside nursing to managing roles: changing occupational perceptions of nursing students. International Journal of Nursing Practice 7, pp. 84-91. 